\title{
Avaliação Econômica de um Serviço de Telemedicina para ampliação da Atenção Primária à Saúde no Rio Grande do Sul: o microcusteio do Projeto TeleOftalmo
}

\author{
Economic Evaluation of a Telemedicine Service to expand Primary \\ Health Care in Rio Grande do Sul: TeleOftalmo's microcosting \\ analysis
}

\author{
Bruna Stella Zanotto (https://orcid.org/0000-0003-4012-1395) ${ }^{1}$ \\ Ana Paula Beck da Silva Etges (https://orcid.org/0000-0002-6411-3480) ${ }^{1}$ \\ Ana Célia Siqueira (https://orcid.org/0000-0003-0703-9023) ${ }^{2}$ \\ Rodolfo Souza da Silva (https://orcid.org/0000-0001-6848-3385) ${ }^{2}$ \\ Cynthia Bastos (https://orcid.org/0000-0002-2504-2915) ${ }^{2}$ \\ Aline Lutz de Araujo (https://orcid.org/0000-0002-6009-1069) ${ }^{2}$ \\ Taís de Campos Moreira (https://orcid.org/0000-0003-0968-9599) ${ }^{3}$ \\ Lucas Matturro (https://orcid.org/0000-0003-1905-6357) ${ }^{3}$ \\ Carisi Anne Polanczyk (https://orcid.org/0000-0002-2447-2577) ${ }^{1}$ \\ Marcelo Gonçalves (https://orcid.org/0000-0001-8516-8547) ${ }^{2}$
}

${ }^{1}$ Instituto Nacional de Ciência e Tecnologia para Avaliação de Tecnologias em Saúde, Universidade Federal do Rio Grande do Sul. R. Ramiro Barcelos 2350, Santa Cecília. 90035-007 Porto Alegre RS Brasil. brunaszanotto@gmail.com ${ }^{2}$ Núcleo Técnico-Científico do Programa Telessaúde Brasil-Redes, Programa de Pós-Graduação em Epidemiologia, Faculdade de Medicina, Universidade Federal do Rio Grande do Sul. Porto Alegre RS Brasil.

${ }^{3}$ Hospital Moinhos de

Vento. Porto Alegre RS

Brasil.

\begin{abstract}
This study evaluated the cost of public telediagnostic service in ophthalmology. The time-driven activity-based costing method (TDA$B C)$ was adopted to examine the cost components related to teleophthalmology. This method allowed us to establish the standard unit cost of telediagnosis, given the installed capacity and utilization of professionals. We considered data from one year of telediagnoses and evaluated the cost per telediagnosis change throughout techno$\log y$ adaptation in the system. The standard cost calculated by distance ophthalmic diagnosis was approximately $R \$ 119$, considering the issuance of 1,080 monthly ophthalmic telediagnostic reports. We identified an imbalance between activities, which suggests the TDABC method's ability to guide management actions and improve resource allocation. The actual unit cost fell from $R \$ 783$ to $R \$ 283$ over one year - with room to approach the estimated standard cost. Partial economic evaluations contribute significantly to support the incorporation of new technologies. The TDABC method deserves prominence, as it enables us to retrieve more accurate information on the cost of technology, improving the scalability and management capacity of the healthcare system.
\end{abstract}

Key words Health Technology Assessment, Telemedicine, Teleophthalmology
Resumo Este estudo avaliou o custo de um serviço público de telediagnóstico em oftalmologia. $O$ método de custeio baseado em atividades e tempo (TDABC) foi adotado para examinar os componentes de custos relacionados à teleoftalmologia. Com este método, também foi possivel estabelecer o custo unitário padrão que o telediagnóstico deveria ter, dada a capacidade instalada e utilização de profissionais. Dados de um ano de telediagnósticos foram considerados, e avaliou-se a mudança do custo por telediagnóstico ao longo do período de adaptação da tecnologia no sistema. O custo padrão calculado por diagnóstico oftalmológico a distância foi de $R \$ 119$, considerando a emissão de 1.080 laudos de telediagnóstico oftalmológico por mês. Foi identificado um desequilíbrio entre as atividades que sugere a capacidade do método TDABC orientar ações de gestão e melhoria na alocação dos recursos. Ao longo de um ano, $o$ custo unitário real passou de $R \$ 783$ para $R \$ 283$, ainda havendo espaço para se aproximar do custo padrão estimado. Avaliações econômicas parciais possuem importante aporte para subsidiar a incorporação de novas tecnologias. O TDABC merece destaque nesse sentido, pois permite obter informações mais precisas sobre custo da tecnologia, melhorando a capacidade de dimensionamento $e$ gerenciamento da organização de saúde.

Palavras-chave Avaliação de Tecnologias em Saúde, Telemedicina, Teleoftalmologia 


\section{Introdução}

Telemedicina pode ser definida como o uso de tecnologias de informação e comunicação na em saúde, viabilizando a oferta de serviços ligados a melhora do acesso do paciente ao cuidado ${ }^{1}$. O surgimento de novas capacidades tecnológicas e sua integração em sistemas de prestação de cuidados em saúde oferece oportunidades para melhorar o atendimento clínico principalmente nos casos em que a distância é um fator crítico ${ }^{1,2}$. Em uma realidade em que os sistemas de saúde estão sob pressão para reduzir custos e, ao mesmo tempo, melhorar a qualidade, acesso e equidade do serviço ${ }^{3}$, ferramentas que se mostrem como uma alternativa frente a este cenário de mudanças precisam ser exploradas.

Preocupações crescentes com a sustentabilidade e escassez prevista de recurso despertam interesse em explorar o potencial da telemedicina para enfrentar muitos desafios enfrentados pela atenção primária à saúde (APS) ${ }^{4}$. Entre os valores associados à adoção de serviços de telemedicina na APS, tem-se a colaboração com o acesso ágil dos pacientes aos centros de alta complexidade ${ }^{5}$, maior resolubilidade no nível básico e diminuição do número de encaminhamentos a outros municípios para atendimento especializado ${ }^{6}$, além de ser um aliado para monitoramento e controle da qualidade de entrega do cuidado ao paciente ${ }^{7}$.

A maioria dos serviços de telemedicina que incide sobre o diagnóstico e manejo clínico já é rotineiramente oferecida nos países mais desenvolvidos, nesse contexto recentes publicações tratam como eficazes esses serviços na gestão de pacientes com doenças crônicas ${ }^{8}$, como por exemplo para realização de laudos de eletrocardiogramas $^{9}$, rastreio de retinopatia diabética ${ }^{10,11}$ e glaucoma ${ }^{12}$, orientação de pacientes através de aplicativos móveis ${ }^{13}$ e reabilitação cardíaca ${ }^{14}$.

O Brasil é um país que oferece oportunidades ímpares para o desenvolvimento e as aplicações da telemedicina dado sua distribuição geográfica e incapacidade de atender a demanda de atendimento presencial ${ }^{2,15,16}$. De acordo com dados do sistema de gerenciamento de consultas ambulatoriais especializadas fornecidos pela Secretaria Estadual da Saúde do Rio Grande do Sul, ao final de 2016 o número de pessoas aguardando por uma consulta especializada em oftalmologia na capital era cerca de 10 vezes mais que a oferta mensal de consultas, representando uma espera de até 19 meses ${ }^{17}$. Tecnologias de telemedicina podem representar um grande avanço na prestação de cui- dados em saúde neste contexto, podendo ter papel estratégico na consolidação de Redes de Atenção à Saúde e melhoria da saúde da população ${ }^{18}$.

Ainda são recentes as avaliações econômicas existentes do assunto e que não permitam uma conclusão assertiva sobre a viabilidade econômica da implementação no Sistema de Saúde Brasileiro ${ }^{19}$. Sabe-se que os investimentos científicos e financeiros necessários para introduzir essas tecnologias no sistema são altos ${ }^{20}$ potencializando a importância da condução de análises econômicas acuradas a fim de orientar decisões de implementação de serviços de telemedicina ${ }^{16,20}$.

Entender a informação de custos é desafiante na avaliação de tecnologias no setor público brasileiro e, embora contraintuitivo, a prática de mensuração de custos não é difundida adequadamente entre os centros de saúde ${ }^{21}$. Um dos motivos é o uso de diferentes metodologias de custeio, da ausência de definição de padrões e da ausência de sistema de custos informatizados nas instituições públicas, o que dificulta a comparabilidade entre diferentes serviços de saúde ${ }^{22}$. A falta de compreensão detalhada do custo real para prestar assistência ao paciente é comumente o problema central de escalabilidade dos gastos com saúde ${ }^{23}$. Nesse sentido, estimativas de microcusteio são o padrão-ouro para valoração de custos pois fornecem um grande grau de detalhamento, principalmente para serviços mais complexos ou que os recursos humanos têm peso maior ${ }^{24}$, como é o caso de serviços de telemedicina.

Alguns métodos de microcusteio utilizados na área da saúde desde a década de 80 são o método de custeio por absorção e o custeio baseado em atividade (Activity-Based Costing - ABC), cujo principal pressuposto é o de corrigir algumas deficiências dos métodos tradicionais, tais como os rateios proporcionais ${ }^{25}$. No entanto, o tradicional modelo $\mathrm{ABC}$ é de difícil execução para muitas organizações, devido às elevadas despesas de execução na entrevista de pessoas, para o inquérito inicial do método $\mathrm{ABC}^{26}$.

Com o intuito de superar essas limitações, Kaplan e Anderson desenvolveram o método de custeio Time-Driven Activity-Based Costing $(\mathrm{TDABC})^{26}$. O TDABC tem o propósito de mensurar o custo dos serviços a partir dos seus reais consumos de recursos. Desde a sua proposição inicial em 2007, vem sendo aplicado em diversos estudos de saúde ${ }^{22,27,28}$. Este método permite identificar os contribuintes individuais e contabilizar os custos indiretos necessários para dar suporte aos cuidados com os pacientes ${ }^{29}$. O princípio fundamental deste método é que ele trans- 
forma os direcionadores de custos em equações de tempo que representam o tempo necessário para se realizar determinada atividade ${ }^{30}$. Outra vantagem do uso de método de microcusteio quando se trata de implementação de tecnologias é que este permite a identificação do custo padrão, isto é, o custo a nível unitário do serviço dentro das condições de eficiência previstas, atuando também como métrica de comparação da produtividade $^{31}$.

Este estudo tem o objetivo de avaliar o custo de um serviço de telemedicina (TelessaúdeRS-UFRGS) utilizando o escopo do telediagnóstico em oftalmologia. Como objetivo específico, tem-se a avaliação do custo de aprendizado relacionado à implantação do serviço na APS.

\section{Métodos}

Por ser uma avaliação econômica parcial, fornecendo informação sobre custo sem fazer referência a efetividade ou comparação entre alternativas, a organização da descrição do estudo foi feita seguindo as conformidades recomendadas pelo Consolidated health economic evaluation reporting standards (CHEERS) ${ }^{32}$.

$\mathrm{O}$ estudo seguiu a estrutura de oito passos sugeridos por Etges et al. ${ }^{22}$ para implementar o custeio baseado em atividades em serviços de saúde: (i) seleção da tecnologia a ser avaliada; (ii) mapeamento do processo do serviço de telemedicina; (iii) identificação dos principais recursos utilizados ao longo do processo; (iv) estimativa do custo total de cada grupo de recursos; (v) estimativa da capacidade de cada recurso e calcular a taxa de custo de capacidade unitária (TCU $\mathrm{R} \$ / \mathrm{h}$ ); (vi) análise das estimativas de tempo para cada recurso usado em uma atividade; (vii) cálculo do custo total do atendimento ao paciente; (viii) análise dos dados de custo.

\section{Seleção da tecnologia a ser avaliada}

O Núcleo de Telessaúde da Universidade Federal do Rio Grande do Sul (TelessaúdeRS-UFRGS) foi selecionado como objeto de estudo de telemedicina ${ }^{15,16}$. Sua escolha se deve ao fato de que o combate a doenças oculares é prioridade para o Organização Mundial de Saúde (OMS) ${ }^{33}$ e por demandar um maior interesse entre os gestores em conhecer o real custo do procedimento. $\mathrm{O}$ TeleOftalmo é um projeto de pesquisa e oferta de serviços em telemedicina que está em andamento desde julho de 2017 no estado do Rio Grande do
Sul (RS), Brasil, que recebe financiamento principal do Ministério da Saúde, através do Programa de Apoio ao Desenvolvimento Institucional do Sistema Único de Saúde (PROADI-SUS). Conta com uma central de comando na sede do TelessaúdeRS-UFRGS e oito salas de exame remoto distribuídas no estado do RS. Os médicos atuantes na APS podem solicitar o serviço aos seus pacientes. Após a avaliação do paciente na sala de exame remoto, um laudo de telediagnóstico é emitido pelo médico oftalmologista ao médico solicitante. Este último então conduzirá o caso do paciente com a ajuda das informações recebidas no laudo.

O horizonte do estudo utilizamos os dados do projeto a partir de setembro de 2017, o que permitiu conduzirmos a análise ao longo de sua implementação e observarmos o impacto do fator aprendizado no custo. Foram avaliados a central de comando de teleoftalmologia, onde os profissionais oftalmologistas atuam, e duas salas de exame remoto, nos municípios de Porto Alegre e Santa Rosa. As duas salas de exame estudadas foram as primeiras implantadas e eram as únicas em funcionamento no momento do início deste estudo.

Mensurar custos reais de tecnologias de saúde é uma importante etapa para identificar a sua eficiência e sequente orientação à tomada de decisão ${ }^{34}$. Dessa forma, a análise foi feita sob perspectiva do sistema de saúde.

\section{Mapeamento do processo do serviço de teleoftalmologia}

O mapeamento da prestação de cuidados detalha o caminho do paciente dentro do serviço oferecido, no qual um ponto inicial e final precisam ser definidos para cada paciente. As principais atividades que fazem parte do fluxo de tratamento e as atividades rotineiras do paciente são identificadas, ilustrando o estado atual de um sistema complexo ${ }^{28,35}$. Essa divisão é importante para o desenvolvimento subsequente de equações de custo e alocação de recursos.

Esta etapa envolveu entrevistas com a coordenadora e a médica oftalmologista acrescidas de observações do serviço em operação. O mapa foi apresentado aos profissionais envolvidos no serviço de teleoftalmologia para validação. Quatro macro atividades foram definidas para embasar a avaliação de custos: regulação da solicitação, contato telefônico para agendamento, avaliação do oftalmologista e coleta de exames (telediagnóstico). 


\section{Identificação dos principais recursos utilizados ao longo do processo}

A partir do detalhamento do processo de operação do serviço de teleoftalmologia, para cada atividade foram elencados os recursos necessários para a sua realização. Os recursos foram classificados como estrutura da sala de exame remoto e estrutura da central de comando de teleoftalmologia, sendo estes compostos por custos fixos e de classe de profissionais (estudantes, médicos e técnicos de enfermagem).

\section{Estimativa do custo total de cada grupo de recursos}

Todos os itens de custo associados a cada grupo de recursos devem ser estimados pelo departamento financeiro do projeto TelessaúdeRS-UFRGS.

$\mathrm{O}$ recurso de estrutura física da central de comando de teleoftalmologia foi valorado a partir da estruturação de uma distribuição de custos fixos do projeto TelessaúdeRS-UFRGS que além do serviço de teleoftalmologia possui outros três serviços de telediagnósticos, além da teleconsultoria e da regulação. Somados aos serviços, o projeto também possui departamentos de apoio e gestão. Para distribuir as contas fixas de remuneração de estudantes, salários energia, depreciação, aluguel e manutenção em um primeiro momento foi considerado como critério de rateio o número de profissionais de qualquer classe alocado a cada departamento. $\mathrm{Na}$ sequência, os custos atribuídos aos departamentos de apoio e gestão foram distribuídos aos departamentos que realizam algum serviço de telediagnóstico, teleconsultoria ou regulação considerando a composição de carga horária atribuída a cada departamento de serviço como direcionador de custos. A soma do custo direto atribuído à central de comando com o absorvido dos departamentos de apoio e gestão representa o custo da estrutura do centro de teleoftalmologia.

O recurso de estrutura da sala de exame remoto foi calculado a partir do levantamento de materiais, profissionais, o custo de estrutura física da secretaria estadual de saúde, onde o atendimento ocorre em cada um dos municípios analisados.

\section{Estimativas da capacidade de cada recurso e calcular a taxa de custo de capacidade unitária $(T C U-R \$ / h)$}

As capacidades foram calculadas em função da disponibilidade de carga horária dos profis- sionais que exercem as atividades e consomem os recursos de profissionais e de estrutura, pois todas demandam a interação humana. As macro atividades de regulação e contato telefônico são efetuadas por estudantes na estrutura do centro de telediagnóstico; a avaliação por oftalmologistas, na central de comando; e a coleta de exames pelo técnico de enfermagem, na sala de exame remoto.

Na sequência, levantou-se com a controladoria financeira do Telessaúde os valores de remuneração destinados aos estudantes envolvidos, salários dos profissionais assalariados e demais contas pagas mensalmente. Esses foram organizados considerando a classificação feita anteriormente das atividades em que atuam os profissionais.

Com a informação de capacidade e de custo mensal dos recursos envolvidos, calcularam-se as taxas de contribuição por unidade de tempo (TCU) em R $\$ / h$ para cada atividade. Aplicando a equação ${ }^{1}$ foi calculada a TCU de cada atividade, na qual o denominador contempla custos dos profissionais e estrutura consumida por cada atividade e a capacidade a carga horária de profissionais que exercem cada atividade.

$$
\mathrm{TCU}=\frac{(\text { Custos fixos e de pessoal) }}{\text { (Capacidade horária dos profissionais) }}
$$

\section{Análise das estimativas de tempo para cada recurso usado em uma atividade}

Os tempos demandados em cada atividade foram estimados a partir de estudos de observação e coleta de tempos e movimentos. Esses estudos podem recorrer a observações in loco acrescida de revisão por um especialista para analisar o tempo real durante o qual diferentes recursos são dedicados a cada atividade ${ }^{36}$.

Para isso, ao longo de duas semanas durante o horário de funcionamento do TeleOftalmo e em turnos alternados foram observadas o volume de produção de cada atividade por hora, convergindo-se para uma média do tempo unitário demandado para uma unidade de atividade.

\section{Cálculo do custo total do atendimento ao paciente}

As equações de custo e tempo foram estruturadas e o cálculo do custo por atendimento foi realizado. Os custos individuais foram estimados em custo padrão meta e o custo real ao longo dos meses de operação do serviço. Para o cálculo do custo por atendimento padrão meta, foi feita uma 
modelagem dos volumes de atendimento que deveriam ser efetuados por mês em cada macro atividade, com base na capacidade horária disponível e a fim de aproximar-se mais da capacidade instalada da realidade do serviço, considerou-se que uma parcela de $20 \%$ da capacidade de cada atividade é destinada a outras atividades ou ociosidade esperada. A parcela de $20 \%$ foi definida em conjunto com a coordenação do serviço que compreende que essa fração do tempo deve ser dedicada a atividades de pesquisa pelos profissionais. Os custos reais foram calculados considerando o volume real de atendimentos por atividade realizado ao longo de um ano de acompanhamento.

\section{Análise dos dados de custo}

Com os custos reais e padrão avaliados, foi feita uma avaliação do balanceamento de capacidade entre as atividades identificando onde há oportunidades de melhor distribuição de profissional e consequente redução de custos unitários. Por fim, foi discutido o impacto do serviço no estado em que está inserido, comparando-se o volume de atendimentos efetuados pelo serviço de teleoftalmologia com a demanda por consultas nas regiões em que o serviço está em operação. Com essa análise é possível mensurar os efeitos de escala de uso sobre os custos desta tecnologia, tendo em vista os custos fixos elevados necessários para introdução da estratégia no sistema.

Os valores estão expressos na moeda brasileira (Real - R\$) e foi feito o acompanhamento de dados de um ano do serviço, no período de setembro de 2017 a setembro de 2018, expressos nos valores não ajustados coletados por mês. Para fins de referência, o preço médio dos dólares internacionais pela taxa de Paridade do Poder de Compra em 2017 foi de 2,013 37 .

\section{Resultados}

\section{Mapeamento do processo}

O processo foi mapeado a partir de entrevistas com coordenadores e executores do "Projeto TeleOftalmo - Olhar Gaúcho", as quais buscaram compreender o fluxo das atividades executadas, profissionais e local envolvidos, e necessidade de uso de recursos específicos. A Figura 1 ilustra as atividades mapeadas.

\section{Estruturação dos dados financeiros de custo por recursos}

Ao conduzir a distribuição de custos, a central de comando de teleoftalmologia consolidou um custo total mensal de $\mathrm{R} \$ 85.000$ sendo destes $\mathrm{R} \$ 10.570$ (12\%) custos de estudantes, $\mathrm{R} \$ 43.980$ (52\%) profissionais assalariados, $\mathrm{R} \$ 3.900(5 \%)$ outros custos fixos e $\mathrm{R} \$ 26.550$ (31\%) absorvidos das áreas de apoio e gestão do núcleo de TelessaúdeRS-UFRGS. Este resultado é importante para o cálculo das taxas de custo unitárias que serão utilizadas nas equações de tempo de mensuração do custo de um telediagnóstico. O custo da sala de exame remoto consolidou $\mathrm{R} \$ 20.600$ mensais.

$\mathrm{Na}$ sequência, levantou-se a capacidade horária mensal dos profissionais que exercem cada atividade para que as TCUs fossem calculadas. Na Tabela 1 estão descritos os resultados de custeio para as etapas do fluxo de atendimento em teleoftalmologia.

\section{Cálculos do custo de um telediagnóstico e análise do balanceamento do serviço}

Os tempos identificados estão expostos na Tabela 2 que, juntamente com as TCUs ajustadas para $\mathrm{R} \$ / \mathrm{min}$, permitem mensurar o custo padrão meta estimados.

A Tabela 2 indica que o valor de custo que deve ser considerado por telediagnóstico é de $\mathrm{R} \$ 119$. Para alcançar esse valor e considerando a capacidade de profissionais, identifica-se que o sistema deve operacionalizar 6.720 regulações, 1.824 ligações, 1.080 avaliações e 422 telediagnósticos por mês em cada um dos consultórios, respeitando os níveis de capacidade existentes.

Ao longo do ano de operação do serviço, em nenhum mês foi registrado esse nível de produção. As Figuras 2, 3 e 4 apresentam a relação de produção dos profissionais e capacidade instalada não utilizada por eles ao longo dos meses e a evolução do custo unitário de cada atividade.

A análise de um ano do serviço estratificada por atividade permite identificar que a atividade que apresenta maior desequilíbrio e capacidade ociosa é exercida pelos bolsistas e representa em média $3 \%$ do custo total. Os profissionais médicos apresentam a atividade que concentra 57\% dos custos e apesar de estarem ao longo do tempo se aproximando do custo alvo, ainda possuem oportunidade de emitir mais laudos. Os profissionais técnicos de enfermagem são os que mais estão próximos de operarem no nível máximo de capacidade disponível. Esses resultados sugerem 


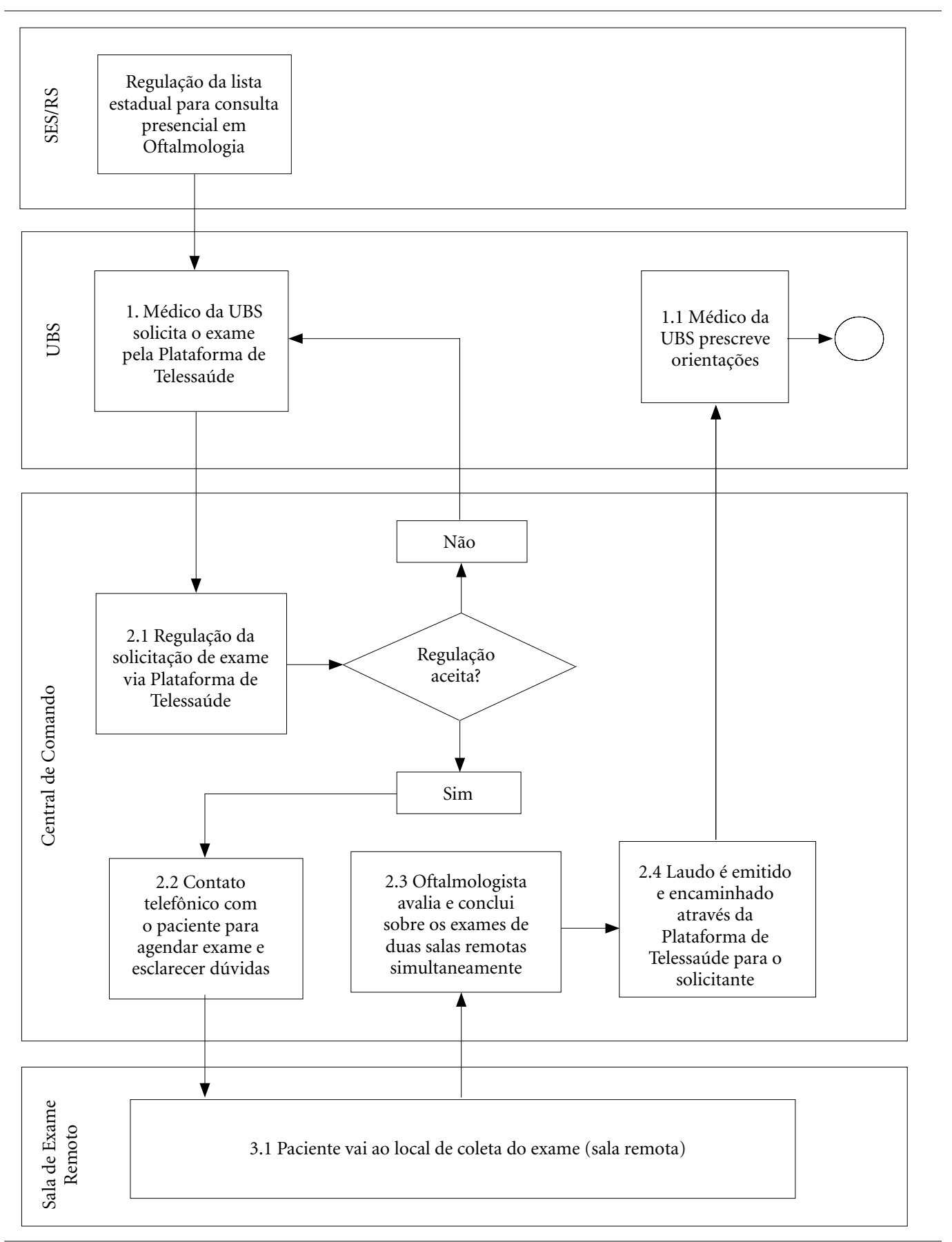

Figura 1. Mapeamento do processo de teleoftalmologia dentro do sistema de atenção primária. SES/RS = Secretaria Estadual de Saúde do Rio Grande do Sul; UBS = Unidade Básica de Saúde.

que há um desequilíbrio entre as capacidades de cada atividade, por exemplo, capacidade de coleta de exames deveria coincidir com a capacidade de emissão de laudos, e através deste método foi possível medir que isto não ocorre no dimensionamento do serviço.
Para fins de avaliação do componente unitário, consolidaram-se então as quatro atividades em um custo individual do serviço de teleoftalmologia, encontrou-se o seguinte comportamento de variação de custos unitários ao longo do tempo (Figura 5). Ao longo de um ano, o custo 
Tabela 1. Custos das atividades relacionados ao atendimento Teleoftalmológico.

\begin{tabular}{|c|c|c|c|c|c|}
\hline Departamento & Atividade & Profissão & $\begin{array}{l}\text { Capacidade } \\
\text { Padrão (h) }\end{array}$ & $\begin{array}{c}\text { Custo } \\
(\mathrm{R} \$)\end{array}$ & $\begin{array}{c}\text { TCU padrão } \\
(\mathrm{R} \$ / \mathrm{h})\end{array}$ \\
\hline \multirow{3}{*}{$\begin{array}{l}\text { Central de comando } \\
\text { de teleoftalmologia }\end{array}$} & Regulação da solicitação & Bolsistas & 112 & 5.490 & 49 \\
\hline & $\begin{array}{l}\text { Contato telefônico com o } \\
\text { paciente }\end{array}$ & Bolsistas & 228 & 10.980 & 48 \\
\hline & Avaliação do oftalmologista & Médicos & 180 & 68.538 & 381 \\
\hline Sala de exame remoto & Coleta de exames & $\begin{array}{l}\text { Técnicos de } \\
\text { enfermagem }\end{array}$ & 422 & 20.606 & 49 \\
\hline
\end{tabular}

TCU = Taxa de Custo Unitário.

Tabela 2. Custo padrão de um telediagnóstico.

\begin{tabular}{lccc}
\hline \multicolumn{1}{c}{ Atividade } & $\begin{array}{c}\text { Tempo } \\
\text { unitário } \\
(\mathbf{m i n})\end{array}$ & $\begin{array}{c}\text { TCU } \\
\text { padrão } \\
(\mathbf{R} \$ \mathbf{m i n})\end{array}$ & $\begin{array}{c}\text { Custo/ } \\
\text { unidade }\end{array}$ \\
\hline $\begin{array}{l}\text { Regulação da } \\
\text { solicitação }\end{array}$ & 1 & 0,82 & $\mathrm{R} \$ 0,80$ \\
$\begin{array}{l}\text { Contato telefônico } \\
\text { para agendamento } \\
\text { Avaliação do }\end{array}$ & 7,5 & 0,8 & $\mathrm{R} \$ 6$ \\
$\begin{array}{l}\text { oftalmologista } \\
\text { Coleta dos exames }\end{array}$ & 60 & 6,35 & $\mathrm{R} \$ 63$ \\
Padrão meta (total) & 78,5 & 0,8 & $\mathrm{R} \$ 49$ \\
\hline
\end{tabular}

TCU = Taxa de Custo Unitário.

unitário apresentou uma amplitude máxima no período de $\mathrm{R} \$ 611$, possuindo na sua majoritariedade uma tendência de aproximação do custo padrão meta estabelecido.

\section{Discussão}

O método aplicado permitiu detalhar os custos associados à adoção de tecnologia de um serviço de telemedicina e revisar a eficácia do seu processo de implementação. O custo aferido padrão por telediagnóstico foi de R \$119. Durante o período de avaliação observou-se uma tendência decrescente do custo unitário do telediagnóstico que foi de $\mathrm{R} \$ 783$ para $\mathrm{R} \$ 283,30$, caracterizando a curva de aprendizado de implementação de novas tecnologias.

Estima-se que a espera por uma consulta para receber prescrição de lentes corretivas é superior a um ano no sul do Brasil ${ }^{16}$. A ausência de critérios racionais no protocolo de agendamento de consultas oftalmológicas, que ocasiona a excessiva demora na prestação do serviço médico, tem como consequência mais grave a perda irreparável da visão, nos casos de glaucoma, retinopatia diabética, entre outras patologias. Para outra parcela significativa de pacientes, com diagnóstico de catarata e refração, a demora implicará em anos de perda substancial de qualidade de vida pela acuidade visual reduzida ${ }^{38-40}$. O advento da telemedicina possui potencial para qualificação da saúde e melhoria do acesso, quando há uma melhor resolubilidade na atenção primária é possível reduzir encaminhamentos para os serviços de atenção secundária e terciária ${ }^{7,38}$.

O comportamento do custo encontrado neste estudo pode ser compreendido como o efeito da aprendizagem sobre a prestação do serviço. A maturidade que pode ser adquirida ao longo do período de aprendizado do serviço contribui para o melhor alinhamento de disponibilidade de profissionais por atividade, tornando o serviço mais equilibrado. $\mathrm{O}$ equilíbrio do serviço permite orientar a reestruturação de disponibilidade horária de profissionais de saúde que exercem o serviço, permitindo uma redução de gastos por telediagnóstico e melhor uso de cada recurso alocado ${ }^{41-43}$.

As avaliações econômicas, começando pela aferição acurada de custos, são grandes aliadas para orientar os decisores políticos e os financiadores a determinar se as evidências apoiam uma adoção mais ampla das intervenções de saúde móvel e entender as condições ideais sob a que eles devem ser implantados ${ }^{24,44}$. Para uma tecnologia ser passível de avaliação, esta deve ser constantemente analisada e aprimorada para que sua adoção ocorra de forma sustentável, transparente e que favoreça sua consolidação no sistema de saúde pública do Brasil ${ }^{34}$. Neste estudo, a avaliação incluiu observação da curva de comportamento da implantação do oferecimento de tal serviço. Torna-se, portanto, clara a necessidade da compatibilização e da integração de dados, permitindo assim um processo contínuo de aprendizagem e aperfeiçoamento das informações sobre o uso do serviço de saúde.

A busca por artigos na literatura mostra que o número de estudos voltados à avaliação de cus- 


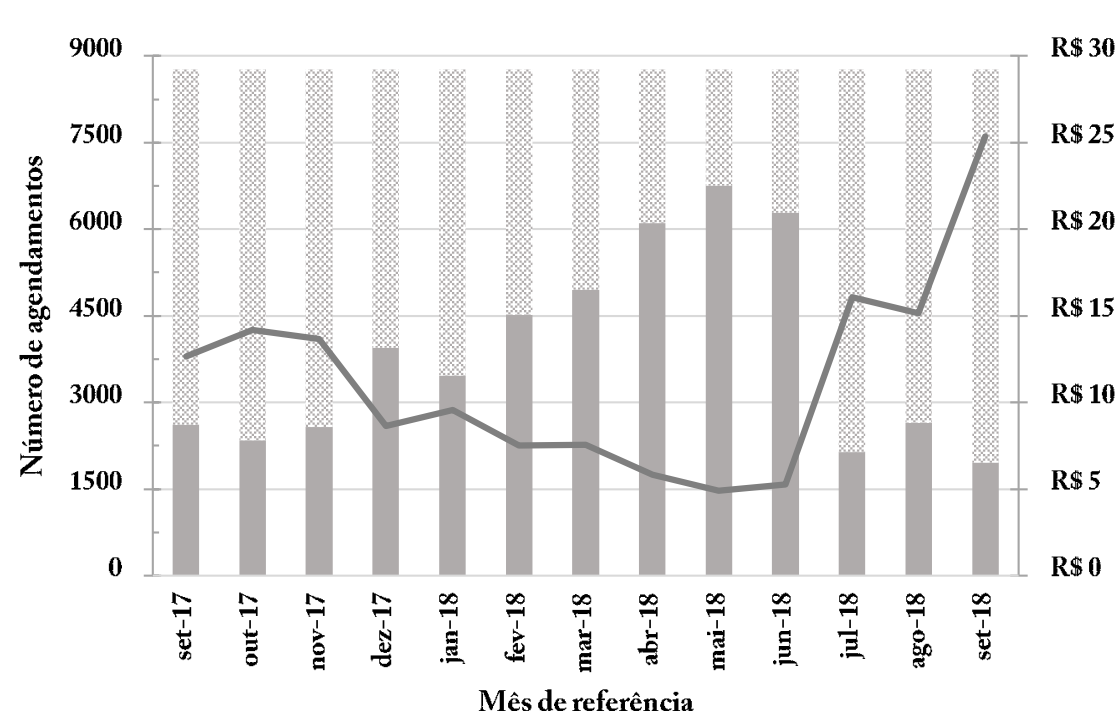

$\$ 30$

$\mathrm{R} \$ 25$

$R \$ 20 \simeq$

$\$ 15$

10

5

$\$ 0$

Capacidade não utilizada profissionais bolsistas

Produção profissionais bolsistas

Custo real unitário

Figura 2. Relação da produção dos estudantes com a capacidade não utilizada para as atividades de regulação e ligação e a evolução do custo unitário das atividades combinadas.

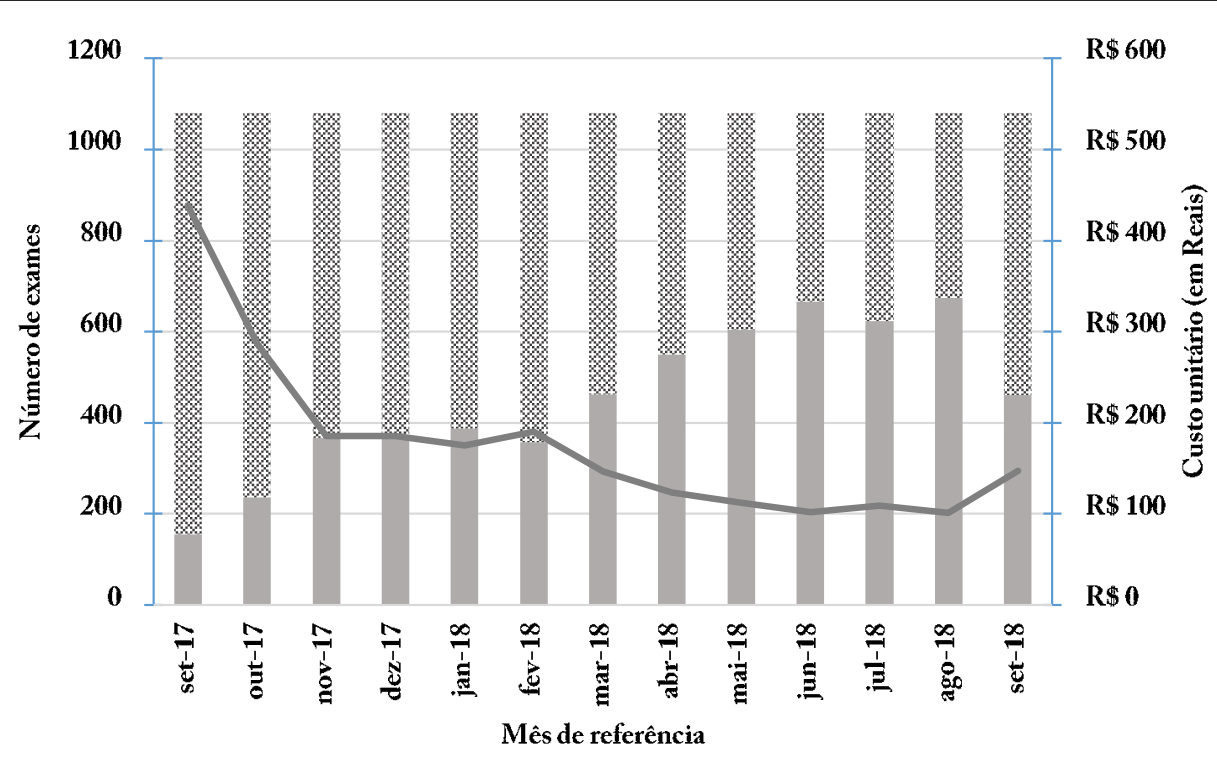

Capacidade não utilizada médicos cotratados

Produção médicos contratados

Custo real unitário

Figura 3. Relação da produção dos médicos com a capacidade não utilizada para as atividades atendimento do oftalmologista e a evolução do custo unitário da atividade. 


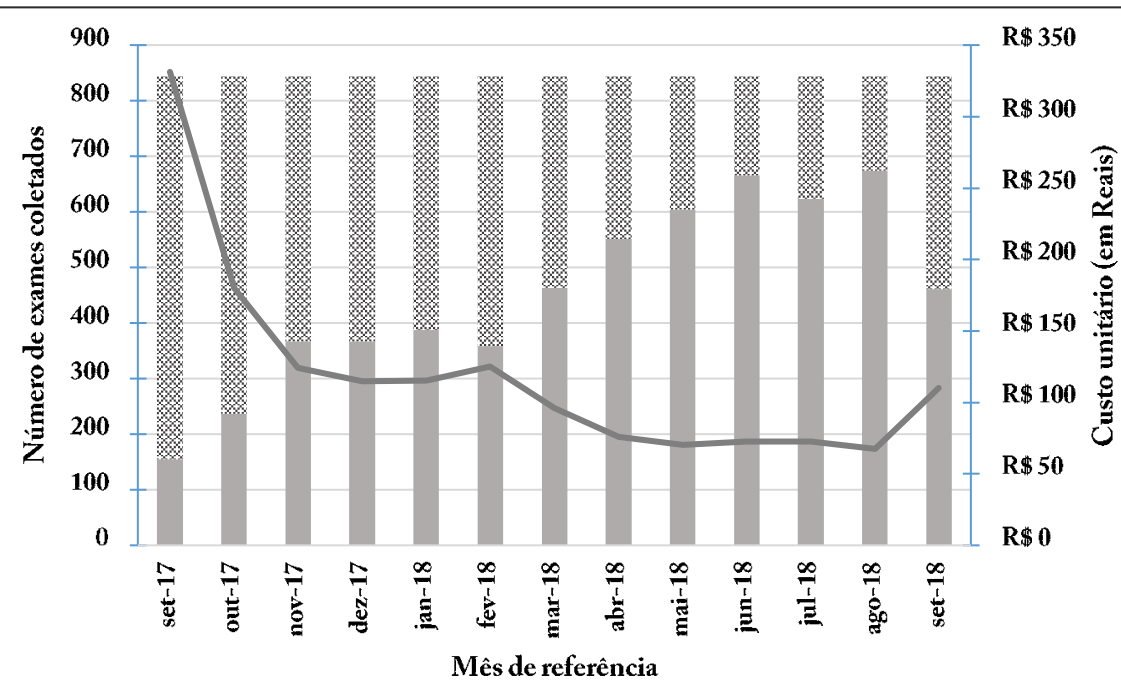

๓⿴囗 Capacidade não utilizada técnicos de enfermagem

Produção técnicos de enfermagem

Custo real unitário

Figura 4. Relação da produção dos técnicos de enfermagem com a capacidade não utilizada para as atividades de coleta de exames e a evolução do custo unitário da atividade.

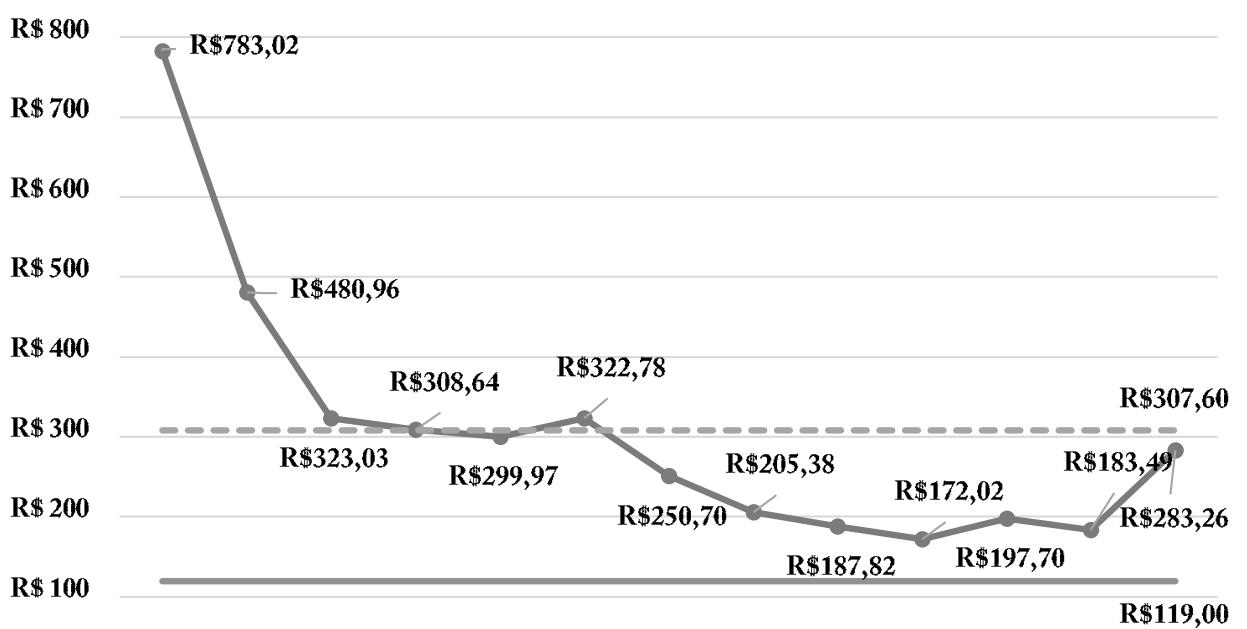

R\$ 0

set/17 out/17 nov/17 dez/17 jan/18 fev/18 mar/18 abr/18 mai/18 jun/18 jul/18 ago/18 set/18

Custo mensal

Meta de custo-padrão

- Média de custo mensal (todo o período da análise)

Figura 5. Custo real do telediagnóstico ao longo de 12 anos pós-implementação. 
tos de serviços de telemedicina aumentou na última década. A origem dos custos é na sua maioria secundária, proveniente de tabelas nacionais, preços estimados e médias. Não se realizou um cálculo pormenorizado do valor investido para instalação e/ou continuidade do programa de telemedicina. $\mathrm{Na}$ área de teleoftalmologia, não foi incomum os autores alegarem que a aferição e detalhamento dos custos se faz necessária para atribuir o benefício indireto da telemedicina no sistema de saúde $\mathrm{u}^{10,11,45}$ no entanto apenas dois deles fizeram uso de métodos de microcusteio na variável custos, ambos específicos para rastreamento de retinopatia diabética. No cenário brasileiro $^{46}$, estudos de implementação e custo-efetividade de telemedicina na área de cardiologia já foram feitos e da mesma forma consideram gastos de implementação separados dos demais, dado o período de adaptação da tecnologia e pessoal envolvidos. No entanto, devido às diferenças de metodologia, tempo de publicação e coleta de dados, as comparações entre os resultados são limitadas e devem ser feitas com cautela.

Dentre as limitações, pode-se citar a não realização de uma avaliação econômica completa, visto que não foram incluídas no estudo métricas de desfecho suficientes para realizar uma avaliação de custo-efetividade ou custo-utilidade. Destaca-se também que o uso desses resultados para fins de comparação com outros Estados e serviços deve considerar que o serviço do Telessaúde é composto por profissionais bolsistas e celetistas e, por isso, consegue operar com menor custo por telediagnóstico.

Esse resultado é relevante para o planejamento da oferta de serviços de saúde no país, visto que mostra que a implantação de sistemas de teleoftalmologia é factível e possui grande potencial para qualificar a fila de espera de atendimento oftalmológico na APS. Com ganho de escala, a telemedicina será essencial para vencer o gargalo das filas para procedimentos de alta complexidade pois possibilita a classificação de risco, qualificando, desta forma, o acesso à especialidade e o próprio cuidado ao usuário ${ }^{47}$.

\section{Conclusões}

Este estudo é inédito no Brasil ao avaliar custos reais de um serviço de telemedicina e acredita-se que a multiplicação de estudos de avaliação econômica parciais destes serviços é essencial para fundamentar decisões de incorporação. Para isso ser possível e escalável, o uso do TDABC merece destaque, pois permite obter informações mais precisas sobre custo da tecnologia, melhorando a capacidade de gerenciamento de organização de saúde. O caso estudado permitiu identificar como a implementação de uma nova tecnologia no sistema de saúde precisa de tempo de maturidade para que se possa mensurar o custo padrão a ser utilizado para orientar a decisão sobre incorporação da tecnologia. Aplicar metodologias que melhor viabilizem a mensuração e análise das informações financeiras passa a ser fundamental para gestão estratégica da saúde e sequente orientação à tomada de decisão. Estudos futuros de custo-efetividade serão realizados como prosseguimento da presente avaliação econômica.

\section{Colaboradores}

BS Zanotto e APBS Etges participaram da coleta de dados e acompanhamento; análise e interpretação dos resultados; e redação do manuscrito. AC Siqueira e C Bastos participaram da coleta de dados e acompanhamento. RS Silva participou da coleta de dados e acompanhamento; e redação do manuscrito. AL Araujo participou da coleta de dados e acompanhamento; e revisão final das análises e manuscrito. TC Moreira, L Maturro e M Gonçalves participaram da revisão final das análises e manuscrito. CA Polanczyk participou da análise e interpretação dos resultados; redação do manuscrito; e revisão final das análises e manuscrito.

\section{Agradecimentos}

O apoio financeiro foi fornecido pelo Ministério da Saúde, por meio do Programa de Apoio ao Desenvolvimento Institucional do SUS (PROADI-SUS), pela Secretaria de Estado da Saúde do Rio Grande do Sul e Instituto Nacional de Ciência e tecnologia para Avaliação de Tecnologias em Saúde (IATS) - CNPq/Brasil. 


\section{Referências}

1. Tuckson RV, Edmunds M, Hodgkins ML. Telehealth. N Engl J Med 2017; 377(16):1585-1592.

2. Maldonado JMSV, Marques AB, Cruz A. Telemedicina: desafios à sua difusão no Brasil. Cad Saude Publica 2016; 32(Supl. 2):e00155615.

3. Halvorsen PA, Kristiansen IS. Radiology services for remote communities: cost minimisation study of telemedicine. BMJ 1996; 312(7042):1333-1336.

4. Bashshur RL, Howell JD, Krupinski EA, Harms KM, Bashshur N, Doarn CR. The Empirical Foundations of Telemedicine Interventions in Primary Care. Telemed E-Health 2016; 22(5):342-375.

5. Lamminen H, Lamminen J, Ruohonen K, Uusitalo $\mathrm{H}$. A cost study of teleconsultation for primary-care ophthalmology and dermatology. $J$ Telemed Telecare 2001; 7(3):167-173.

6. Hjelm NM. Benefits and drawbacks of telemedicine. $J$ Telemed Telecare 2005; 11(2):60-70.

7. Flodgren G, Rachas A, Farmer AJ, Inzitari M, Shepperd S. Interactive telemedicine: effects on professional practice and health care outcomes. Cochrane Database Syst Rev 2015; 9:CD002098.

8. Grustam AS, Severens JL, van Nijnatten J, Koymans R, Vrijhoef HJM. Cost-effectiveness of telehealth interventions for chronic heart failure patients: a literature review. Int J Technol Assess Health Care 2014; 30(1):59-68.

9. Kotb A, Cameron C, Hsieh S, Wells G. Comparative Effectiveness of Different Forms of Telemedicine for Individuals with Heart Failure (HF): A Systematic Review and Network Meta-Analysis. PLoS One 2015; 10(2): 0118681

10. Rachapelle S, Legood R, Alavi Y, Lindfield R, Sharma T, Kuper H, Polack S. The Cost-Utility of Telemedicine to Screen for Diabetic Retinopathy in India. Ophthalmology 2013; 120(3):566-573.

11. Castillo-Riquelme MC, Lord J, Moseley MJ, Fielder AR, Haines L. Cost-effectiveness of digital photographic screening for retinopathy of prematurity in the United Kingdom. Int J Technol Assess Health Care 2004; 20(2):201-213.

12. Thomas S, Hodge W, Malvankar-Mehta M. The Cost-Effectiveness Analysis of Teleglaucoma Screening Device. PLoS One 2015; 10(9):e0137913.

13. Rush KL, Hatt L, Janke R, Burton L, Ferrier M, Tetrault M. The efficacy of telehealth delivered educational approaches for patients with chronic diseases: A systematic review. Patient Educ Couns 2018; 101(8):1310-1321.

14. Hamilton SJ, Mills B, Birch EM, Thompson SC. Smartphones in the secondary prevention of cardiovascular disease: a systematic review. BMC Cardiovasc Disord 2018; 18(1):25.

15. Harzheim E, Gonçalves MR, Umpierre RN, Siqueira ACS, Katz N, Agostinho MR, Oliveira EB, Basso J, Roman R, Moro RG, Pilz C, Heinzelmann RS, Schmitz CAA, Hauser L, Mengue SS. Telehealth in Rio Grande do Sul, Brazil: Bridging the Gaps. Telemed E-Health 2016; 22(11):938-944
16. Gonçalves MR, Umpierre RN, D'Avila OP, Katz N, Mengue SS, Siqueira ACS, Carrard VC, Schmitz CAA, Molina-Bastos CG, Rados DV, Agostinho MR, Oliveira EB, Roman R, Basso J, Pfeil JN, Mendonça MVA, Moro RG, Frank T, Stürmer PL, Harzheim E. Expanding Primary Care Access: A Telehealth Success Story. Ann Fam Med 2017; 15(4):383.

17. Secretaria de Saúde Estadual. Gestão e Tecnologia em Saúde Sistema AGHOS. 2016 (não publicado).

18. Brasil. Ministério da Saúde (MS), Secretaria de Ciência, Tecnologia e Insumos Estratégicos, Departamento de Ciência e Tecnologia. Guia Metodológico para Programas e Serviços em Telessaúde [documento na Internet]. Brasilia: MS; 2019 [acessado 2019 Set 4]. Disponível em: https://www.telessaude.unifesp.br/ images/docs/Guia_Monitoramento_Telessaude_ WEB.pdf

19. Lin M, Yuan W, Huang T, Zhang H, Mai J, Wang J. Clinical effectiveness of telemedicine for chronic heart failure: a systematic review and meta-analysis. $J$ Investig Med 2017; 65(5):899-911.

20. Iribarren SJ, Cato K, Falzon L, Stone PW. What is the economic evidence for mHealth? A systematic review of economic evaluations of mHealth solutions. PLoS One 2017; 12(2):e0170581.

21. Souza RJSP, Mattedi AP, Rezende ML, Corrêa MP, Duarte EM. Estimativa do custo do tratamento de câncer de pele tipo melanoma no Estado de São Paulo - Brasil. An Bras Dermatol 2009 ;84(3):237-343.

22. Etges APBS, Cruz LN, Notti RK, Neyeloff JL, Schlatter RP, Astigarraga CC, Falavigna M, Polanczyk CA. An 8-step framework for implementing time-driven activity-based costing in healthcare studies. Eur J Health Econ 2019; 20(8):1133-1145.

23. Martin JA, Mayhew CR, Morris AJ, Bader AM, Tsai $\mathrm{MH}$, Urman RD. Using Time-Driven Activity-Based Costing as a Key Component of the Value Platform: A Pilot Analysis of Colonoscopy, Aortic Valve Replacement and Carpal Tunnel Release Procedures. J Clin Med Res 2018; 10(4):314-320.

24. Brasil. Ministério da Saúde (MS), Secretaria de Ciência, Tecnologia e Insumos Estratégicos, Departamento de Ciência e Tecnologia. Diretrizes metodológicas : Diretriz de Avaliação Econômica. Brasília: MS; 2014.

25. Kaplan RS, Cooper R. Custo e desempenho: administre seus custos para ser mais competitivo. São Paulo: Futura; 1998.

26. Kaplan RS, Anderson SR. The Innovation of Time-Driven Activity-Based Costing. J Cost Manag 2007; 21(2):5-15.

27. Keel G, Savage C, Rafiq M, Mazzocato P. Time-driven activity-based costing in health care: A systematic review of the literature. Health Policy 2017; 121(7):755763.

28. Erhun F, Mistry B, Platchek T, Milstein A, Narayanan VG, Kaplan RS. Time-driven activity-based costing of multivessel coronary artery bypass grafting across national boundaries to identify improvement opportunities: study protocol. BMJ 2015; 5(8):e008765. 
29. Chapko MK, Liu C-F, Perkins M, Li Y-F, Fortney JC, Maciejewski ML. Equivalence of two healthcare costing methods: bottom-up and top-down. Health Econ 2009; 18(10):1188-1201.

30. Heaton HA, Nestler DM, Barry WJ, Helmers RA, Sir MY, Goyal DG, Haas DA, Kaplan RS, Sadosty AT. A Time-Driven Activity-Based Costing Analysis of Emergency Department Scribes. Mayo Clin Proc Innov Qual Outcomes 2019; 3(1):30-34.

31. Martins E. Contabilidade de custos. São Paulo: Atlas; 2008.

32. Husereau D, Drummond M, Petrou S. Consolidated health economic evaluation reporting standards (CHEERS) - Explanation and elaboration: A report of the ISPOR health economic evaluations publication guidelines good reporting practices task force. Value Health 2013; 16(2):231-250.

33. Dua HS, Singh AD. Global estimates of visual impairment: 2010. Br J Ophthalmol 2012; 96(5):614-618.

34. Lima SGG, Brito C, Andrade CJC. Health technology assessment in Brazil - an international perspective. Cien Saude Colet 2019; 24(5):1709-1722.

35. Golini R, Kalchschmidt M, Landoni P. Adoption of project management practices: The impact on international development projects of non-governmental organizations. Int J Proj Manag 2015; 33(3):650-663.

36. Kaplan RS, Witkowski ML, Hohman JA. Children's Hospital Boston: measuring patient costs. C. R. Math 2012; 3(1):e28.

37. Sistema Gerenciador de Séries Temporais (SGS) [página na Internet]. [acessado 2019 Set 4]. Disponível em: https://www3.bcb.gov.br/sgspub/consultarvalores /consultarValoresSeries.do? method=consultarValores

38. Tahhan N, Papas E, Fricke TR, Frick KD, Holden BA. Utility and Uncorrected Refractive Error. Ophthalmology 2013; 120(9):1736-1744.

39. Cypel MC, Salomão SR, Dantas PEC, Lottenberg CL, Kasahara N, Ramos LR, Belfort Junior R. Vision status, ophthalmic assessment, and quality of life in the very old. Arq Bras Oftalmol 2017; 80(3):159-164.

40. GBD 2017 DALYs HALE Collaborators. Global, regional, and national disability-adjusted life-years (DALYs) for 359 diseases and injuries and healthy life expectancy (HALE) for 195 countries and territories, 1990-2017: a systematic analysis for the Global Burden of Disease Study 2017. Lancet 2018; 392(10159):1859-1922.
41. McLaughlin N, Garrett MC, Emami L, Foss SK, Klohn JL, Martin NA. Integrating risk management data in quality improvement initiatives within an academic neurosurgery department. J Neurosurg 2016; 124(1):199-206.

42. French KE, Albright HW, Frenzel JC, Incalcaterra JR, Rubio AC, Jones JF, Feeley TW. Measuring the value of process improvement initiatives in a preoperative assessment center using time-driven activity-based costing. Healthc (Amst) 2013; 1(3-4):136-142.

43. Koehler DM, Balakrishnan R, Lawler EA, Shah AS, Endoscopic Versus Open Carpal Tunnel Release: A Detailed Analysis Using Time-Driven Activity-Based Costing at an Academic Medical Center. J Hand Surg 2019; 44(1):62.e1-62.e9.

44. Le Goff-Pronost M, Sicotte C. The added value of thorough economic evaluation of telemedicine networks. Eur J Health Econ 2010; 11(1):45-55.

45. Nguyen HV, Tan GSW, Tapp RJ, Mital S, Ting DSW, Wong HT, Tan CS, Laude A, Tai ES, Tan NC, Finkelstein EA, Wong TY, Lamoureux EL. Cost-effectiveness of a National Telemedicine Diabetic Retinopathy Screening Program in Singapore. Ophthalmology 2016; 123(12):2571-2580.

46. Andrade MV, Maia AC, Cardoso CS, Alkmim MB, Ribeiro ALP. Custo-Benefício do Serviço de Telecardiologia no Estado de Minas Gerais: Projeto Minas Telecardio. Custo-benefício da telecardiologia 2011; 97:307-316.

47. Maeyama MA, Calvo MCM. A Integração do Telessaúde nas Centrais de Regulação: a Teleconsultoria como Mediadora entre a Atenção Básica e a Atenção Especializada. Rev Bras Educ Médica 2018; 42(2):6372.

Artigo apresentado em 03/10/2019

Aprovado em 21/10/2019

Versão final apresentada em 23/10/2019 